\title{
Attenuation of the Aggregation and Neurotoxicity of Amyloid Peptides with Neurotransmitter-Functionalized Ultra-Small-Sized Gold Nanoparticles
}

\author{
Yueling Xu, ${ }^{1}$ Yiliang Li, ${ }^{2}$ Lin Wei, ${ }^{3}$ Hua Liu, ${ }^{1}$ Juhui Qiu ${ }^{4}$ and Lehui Xiao ${ }^{1, *}$
}

\begin{abstract}
Alzheimer's disease $(\mathrm{AD})$ is closely associated with the self-aggregation of beta-amyloid peptides $(\mathrm{A} \beta)$. The neuron cytotoxicity of assembled $\mathrm{A} \beta$ fibrils has been extensively studied. In this work, ultra-small-sized gold nanoparticles (USGNPs, with diameter of $\sim 4 \mathrm{~nm}$ ) are synthesized and conjugated with neurotransmitter dopamine (DA) and its synthetic precursors (L-phenylalanine (L-Phe) and L-tyrosine (L-Tyr)). It is found that the fibrillation process of $\mathrm{A} \beta$ can be efficiently inhibited by these functionalized USGNPs, which is essentially ascribed to the screened growth points on the short seeds after being anchored by functionalized USGNPs as well as the disturbing of the peptide folding process in solution as revealed by the transmission electron microscope (TEM) and circular dichroism (CD) spectroscopic measurements. More interestingly, the neuron cells (PC12 as a model) exhibit noticeably improved viability $(\sim 90 \%)$ when treated with functionalized USGNPs in contrast to the control treated with $\mathrm{A} \beta$ growth solution alone $(\sim 70 \%)$. Because of the superior inhibition effect on the $\mathrm{A} \beta$ fibrillation as well as the remarkable cell protection effect, these functionalized USGNPs would afford insight information for the rational design of efficient strategy for AD treatment.
\end{abstract}

Keywords: Gold nanoparticles; Amyloid peptide; Fibrillation, Alzheimer's disease; Fluorescence imaging

Received 15 January 2019, Accepted 21 February 2019

DOI: $10.30919 / \mathrm{es} 8 \mathrm{~d} 778$

\section{Introduction}

Alzheimer's disease (AD), as a progressive and irreversible neurodegenerative disease, is characterized by the deposition of $\mathrm{A} \beta$ plaques resulted from the aggregation of $\mathrm{A} \beta$ peptides. The $\mathrm{A} \beta$ peptides mainly consist of 40 and 42 residues (i.e., $\mathrm{A} \beta_{1-40}$ and $\mathrm{A} \beta_{1-42}$ respectively), which are formed through the cleavage of the transmembrane amyloid precursor protein expressed by $\beta$ - and $\gamma$-secretases in the central nervous system. ${ }^{1,2}$ When the balance between the production and clearance of $\mathrm{A} \beta$ fibrils is upset, it undergoes conformation transition from random coil to an $\alpha$-helix intermediate and finally to $\beta$-sheet rich structure accompanied by the gradual accumulation process from monomeric $\mathrm{A} \beta$, soluble oligomeric intermediates to insoluble amyloid fibrils. ${ }^{1}$ These aggregated species have been demonstrated to be neurotoxic, ${ }^{3}$ which disturb the normal function of the brain by changing

State Key Laboratory of Medicinal Chemical Biology, Tianjin Key Laboratory of Biosensing and Molecular Recognition, College of Chemistry, Nankai University, Tianjin, 300071, China

Department of Neurology, Postdoctoral Research Workstation of Clinical Medicine, The Third Xiangya Hospital, Central South University, Changsha 410013, China

Key Laboratory of Chemical Biology \& Traditional Chinese Medicine Research, Key Laboratory of Phytochemical R\&D of Hunan Province, College of Chemistry and Chemical Engineering, Hunan Normal University, Changsha, 410082, China

Key Laboratory for Biorheological Science and Technology of Ministry of Education, State and Local Joint Engineering Laboratory for Vascular Implants, Bioengineering College of Chongqing University, Chongqing, 400030, China

*E-mail:lehuixiao@nankai.edu.cn the synaptic plasticity, ${ }^{4}$ deregulation of $\mathrm{Ca}^{2+}$ homeostasis and result in the gradual synaptic loss, ${ }^{5}$ neuronal death, and cognitive decline. ${ }^{6}$ On this basis, exploring efficient methods to regulate the self-aggregation process of $\mathrm{A} \beta$ has aroused great attention in diverse areas. ${ }^{7.15}$

Until now, plenty of researches have focused on developing effective fibril inhibitors and $\beta$-sheet breakers to disturb the aggregation of $\mathrm{A} \beta$ or dissociate the preformed $\mathrm{A} \beta$ fibrils. ${ }^{11,16}$ For instance, polyphenols and antioxidants such as vitamin $\mathrm{E}$ can inhibit $\mathrm{A} \beta$ fibrillation to some extent. ${ }^{17}$ Metal complexes have also been reported as potent regulators on $\mathrm{A} \beta$ aggregation like zinc (II)-conjugated complexes, binuclear ruthenium (II)-platinum (II) conjugated complexes and so on. ${ }^{18,19}$ Besides, some straightforward evidences verified that the conformation change of $\mathrm{A} \beta$ after adsorption onto the surface of nanoparticles may influence its biological nature, and modulate the aggregation behavior accordingly. ${ }^{20,21}$ For example, poly(acrylic acid) modified $30 \mathrm{~nm}$ gold nanoparticles have been demonstrated with strong inhibition effect on $\mathrm{A} \beta$ fibrillation. ${ }^{22}$ As a consequence, nanomaterialbased approaches provide a more promising platform for the treatment of $\mathrm{AD}$ owing to their unique optical, electrical, catalytic properties, easy of size and shape control and versatile surface chemistry., 16,22-30 For example, Kogan et al. showed the possibility to remotely redissolve $\mathrm{A} \beta$ deposits and to interfere with their growth relying on the local heat delivered by metallic nanoparticles. ${ }^{16}$ Several studies also demonstrated that the thickness and surface charge of the coating layer on nanoparticles could affect the kinetics of fibrillation of $\mathrm{A} \beta$ in vitro. ${ }^{22,23,26,31}$

Interestingly, it has been found that severe loss of memory and cognitive functions, the typical symptoms of $\mathrm{AD}$, are closely related to the change or imbalance of neurotransmitters, which are also known as chemical messengers. ${ }^{32}$ They are released from synaptic vesicles in synapses and enter into the synaptic cleft, where they are received by receptors on the target cells. ${ }^{33}$ Particularly, previous works have shown that the dysfunction in the dopaminergic system may account for the 
symptoms of apathy in $\mathrm{AD}{ }^{34}$ Dopamine (DA), an organic chemical of the catecholamine and phenethylamine families, plays several important roles in the brain and body. In the brain, it can send signals to other nerve cells in order to regulate motor behavior, pleasures related to motivation and emotional arousal. Some evidences have indicated that the intake of neurotransmitter precursors does improve neurotransmitter synthesis. ${ }^{35}$ In the brain, L-Phe is biologically converted into L-Tyr, and then to levodopa, which is further converted into DA, norepinephrine (noradrenaline), and epinephrine (adrenaline). The latter three are known as the catecholamine.

In the $\mathrm{AD}$ treatment, another critical issue is the capability of crossing the blood-brain barrier (BBB). Amount of studies have demonstrated that the $\mathrm{BBB}$ crossing efficiency can be improved as long as the size of nanoparticles is small enough..$^{36-38}$ On this consideration, in this work, we synthesized ultra-small-sized gold nanoparticles (USGNPs) with diameter $\sim 4 \mathrm{~nm}$ and modified them with DA (and its precursor molecules L-Phe and L-Tyr) to explore the potential fibrillation inhibition effect (Scheme 1). With respect to L-Phe and LTyr, they are two amino acids as the building blocks of proteins for both structural and metabolic processes, and the precursors required for the synthesis of DA. Meanwhile, they can cross the BBB. ${ }^{39}$ The realtime fluorescence microscopic and spectroscopic characterizations demonstrated that the $\mathrm{A} \beta_{140}$ fibrillation process in biological milieu can be effectively and specifically regulated by those functionalized USGNPs, which is further confirmed by the comprehensive control experiments with either bare USGNPs or those functional small molecules. The superior inhibition effect of the functionalized USGNPs is basically ascribed to the specific and strong association with the seed fibrils as revealed by the transmission electron microscope (TEM) and circular dichroism (CD) spectroscopic measurements. More interestingly, these functionalized USGNPs are biocompatible to PC12 cell (commonly used as a model system for AD exploration and has been used to get more information about diseases of the brain). The cytotoxic effect of $\mathrm{A} \beta_{1-40}$ aggregates can also be alleviated by introducing those functionalized USGNPs together as proved by the MTT assay and confocal microscopic characterizations. As a consequence, because of the excellent inhibition effect on the $\mathrm{A} \beta_{140}$ fibrillation process, these biocompatible USGNPs will find promising applications in the exploration of robust strategies for the treatment of $\mathrm{AD}$ in the future.

\section{Experimental section}

\subsection{Materials}

$\mathrm{A} \beta_{1-40}(91.0 \%)$ was obtained from Ana Spec (San Jose, CA, U.S.A). $\mathrm{K}_{2} \mathrm{HPO}_{4}(99.0 \%), \mathrm{KH}_{2} \mathrm{PO}_{4}(99.5 \%), \mathrm{Na}_{2} \mathrm{~B}_{4} \mathrm{O}_{7}(99.5 \%), \mathrm{H}_{3} \mathrm{BO}_{3}(99.5 \%)$, thioglycolic acid (95.0\%), ammonia aqueous solution (25-28\%) and thioflavin T (ThT) were obtained from Sigma-Aldrich (St. Louis, MO, U.S.A). N-Hydroxysuccinimide (NHS, 98.0\%), N-(3-Dimethylaminopropyl)-N'ethylcarbodiimide hydrochloride (EDC, 98.5\%), dopamine hydrochloride (DA, 98.0\%), L-phenylalanine (L-Phe, 99.0\%), Ltyrosine (L-Tyr, 99.0\%), dimethyl sulfoxide (DMSO, 99.0\%), $\mathrm{NaBH}_{4}$ (96.0\%) and $\mathrm{HAuCl}_{4}(47.8 \%)$ were obtained from Aladdin (Shanghai, China). Thiazolyl blue tetrazolium bromide (MTT), propidium iodide (PI) and 4', 6-diamidino-2-phenylindole (DAPI) were obtained from Beyotime (China). Fetal bovine serum (FBS) and Dulbecco's modified eagle medium (DMEM) were purchased from Invitrogen Corp (Carlsbad, CA, U.S.A).

2.2 Synthesis and modification of USGNPs with DA, L-Phe and L-Tyr Aqueous solutions of $\mathrm{HAuCl}_{4}(30 \mu \mathrm{L}, 24.28 \mathrm{mM})$, HS-CH$-\mathrm{COOH}(15$ $\left.\mu \mathrm{L}, 1.0 \times 10^{-5} \mathrm{M}\right)$ and $\mathrm{PEG}-\mathrm{CH}_{2}-\mathrm{SH}\left(5 \mu \mathrm{L}, 1.42 \times 10^{-5} \mathrm{M}\right)$ were added to $1.9 \mathrm{~mL}$ of deionized water. After $2 \mathrm{~min}$ of vigorous stirring, $\mathrm{NaBH}_{4}(60$ $\mu \mathrm{L}, 0.15 \mathrm{M})$ was added to the reaction mixture until obtaining brown
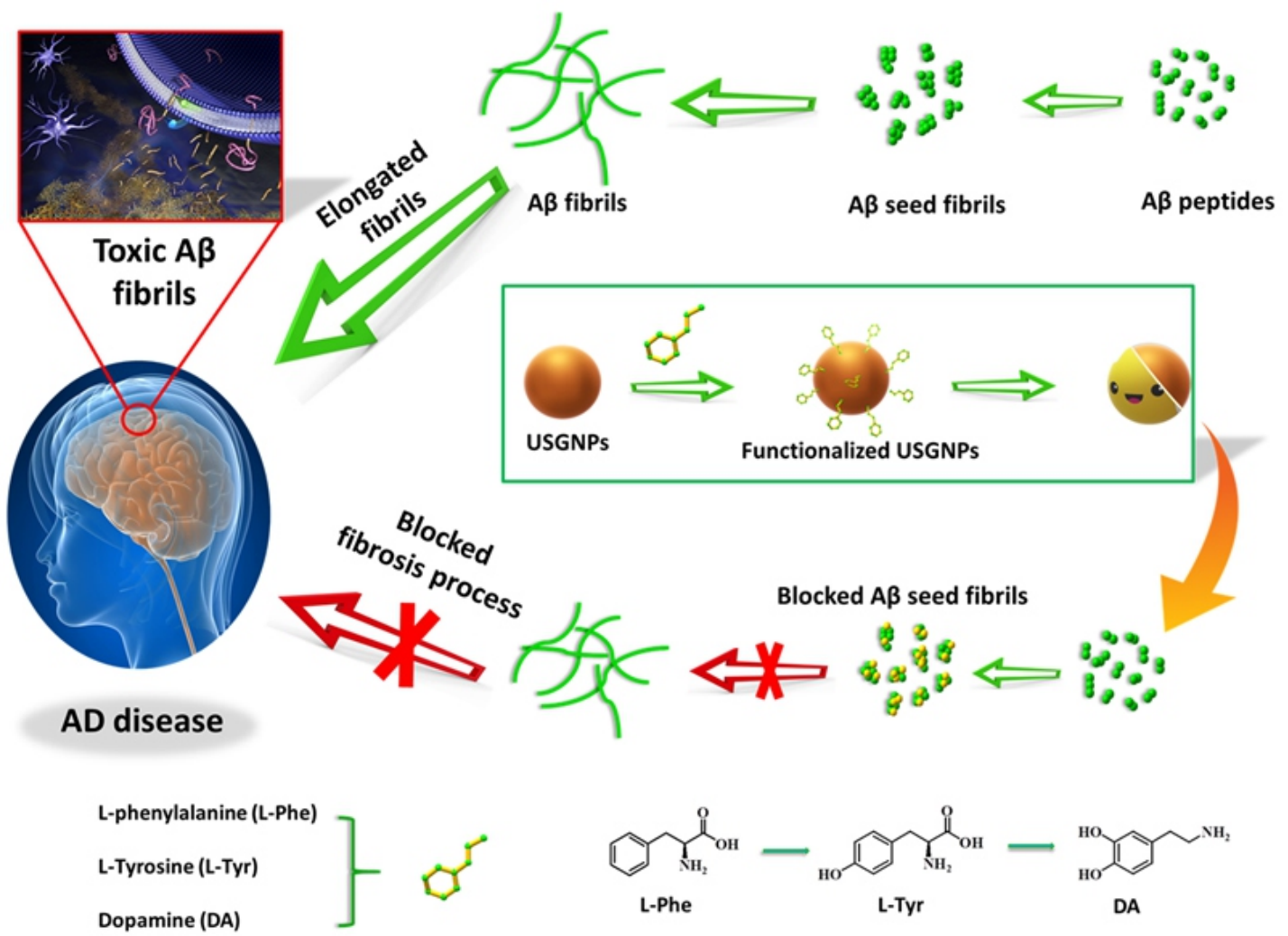

Scheme 1 Schematic diagram of the functionalization process of USGNPs and the modulation of A $\beta_{140}$ fibrillation process with functionalized USGNPs. 
solution. Then the particles were purified by ultrafiltration centrifugation $(4660 \times \mathrm{g})$ for three times. Finally, the solution was stored at $4{ }^{\circ} \mathrm{C}$ before use. The method to synthesize functionalized USGNPs is based on the EDC/NHS crosslinking reaction. Firstly, EDC $\left(1.5 \times 10^{-4} \mathrm{~mol}\right)$ and HS$\mathrm{CH}_{2}-\mathrm{COOH}\left(1.5 \times 10^{-5} \mathrm{~mol}\right)$ were added to $400 \mu \mathrm{L}$ borate buffer $(\mathrm{pH} 8.0$, $50 \mathrm{mM})$ and the mixture was activated for $30 \mathrm{~min}$. Then NHS $\left(3 \times 10^{-4}\right.$ $\mathrm{mol})$ and DA $\left(1.5 \times 10^{-5} \mathrm{~mol}\right)$ were introduced to the mixture and kept stirring (100 rpm) for $5 \mathrm{~h}$. Subsequently, the functionalized DA $(15 \mu \mathrm{L}$, $\left.1.42 \times 10^{4} \mathrm{M}\right)$ and aqueous solutions of $\mathrm{HAuCl}_{4}(30 \mu \mathrm{L}, 24.28 \mathrm{mM})$ were added to $1.9 \mathrm{~mL}$ of deionized water. After $2 \mathrm{~min}$ of vigorous stirring, $\mathrm{NaBH}_{4}(60 \mu \mathrm{L}, 0.15 \mathrm{M})$ was added to the reaction system until obtaining orange solution. Then, the DA-functionalized USGNPs were further purified by ultrafiltration centrifugation three times. The L-Pheand L-Tyr-functionalized USGNPs were also acquired and purified accordingly. The concentration of USGNPs was determined by the UVVis absorption spectroscopy according to Lambert-Beer law. The hydrodynamic size and zeta potential distribution of nanoparticles in solution was characterized by dynamic light scattering (DLS) (Zetasizer Nano-ZS90, U.K.).

\section{$2.3 \mathrm{~A} \boldsymbol{\beta}_{1-40}$ fibrillation and inhibition}

The stock solution of $\mathrm{A} \beta_{140}$ peptide was prepared by dissolving $1 \mathrm{mg}$ $\mathrm{A} \beta_{140}$ peptide powder in $400 \mu \mathrm{L} 0.2 \%$ ammonia aqueous solution without any purification and stored at $-20{ }^{\circ} \mathrm{C}$ until using. The $\mathrm{A} \beta_{140}$ growth solution was prepared by diluting the $\mathrm{A} \beta_{1-40}$ stock solution into $50 \mu \mathrm{M}$ with filtered phosphate buffer ( $\mathrm{PB}, \mathrm{pH} 7.4,25 \mathrm{mM})$. The growth solution was incubated at $37{ }^{\circ} \mathrm{C}$ for different time. In the inhibition assay, the $\mathrm{A} \beta_{1-40}$ growth solution was co-cultured with USGNPs (43.6 $\mathrm{nM})$, free DA $(1.53 \mu \mathrm{M})$, L-Phe $(1.53 \mu \mathrm{M})$, L-Tyr $(1.53 \mu \mathrm{M})$ and corresponding functionalized USGNPs $(43.6 \mathrm{nM})$ respectively. In the concentration dependent inhibition assay with functionalized USGNPs, different concentrations of functionalized USGNPs (ranging from 14.6 to $43.6 \mathrm{nM}$ ) were added to the mixture solution and incubated at $37{ }^{\circ} \mathrm{C}$.

\subsection{Spectroscopic and microscopic characterization}

For the Fourier transform infrared (FT-IR) spectroscopic measurements, the samples were dripped on the potassium bromide sheet and dried with infrared lamp. The spectra were measured on a Nicolet Avatar 370 instrument (Thermo Fisher Scientific, Inc., Waltham, MA). The circular dichroism (CD) spectra were determined by a JASCO J-720 CD spectrometer instrument (Jasco Co., Tokyo, Japan). Each sample was repeated three times. The ThT fluorescence spectroscopic measurements were performed on a Hitachi F-2500 fluorescence spectrophotometer (Hitachi, Ltd., Tokyo, Japan) by mixing the $\mathrm{A} \beta_{140}$ growth solution with ThT in the concentration ratio 1:1. For the TEM characterization, diluted sample solution of $4 \mu \mathrm{L}$ was dropped on a carbon-coated copper grid and blotted with filter paper. The sample was then negatively stained with $2 \%$ uranyl acetate. The dried sample was examined by a JEM 1230 TEM (JEOL, Japan) with an acceleration voltage of $210 \mathrm{kV}$ for high-resolution TEM images. The fluorescence microscopic imaging experiments of the amyloid fibrils were performed on a Nikon Ti-U inverted fluorescence microscope (Nikon, Japan) under total internal reflection fluorescence (TIRF) imaging mode. The fluorescence from the ThT labelled $\mathrm{A} \beta_{1-40}$ was collected by a $100 \times$ objective (NA 1.49) and recorded with an EMCCD (ultra-897, Andor, U.K.). All of the data recorded by the EMCCD camera were analyzed by the public image processing software, Image J (http://rsbweb.nih.gov/ij/).

\subsection{MTT cytotoxicity assay}

Pc12 cells were incubated in 96-well plates at a density of $\sim 5000$ cells/well in DMEM containing $10 \%$ FBS and incubated at $37{ }^{\circ} \mathrm{C}$ for
$24 \mathrm{~h}$. Then, the medium was replaced with the fresh medium containing the solution of the corresponding samples (e.g., different concentrations of functionalized USGNPs, the end-point products of $\mathrm{A} \beta_{140}$ growth solution and so on). After $24 \mathrm{~h}$ co-incubation, the medium was removed and the cells were washed several times with PBS. Then $20 \mu \mathrm{L}$ of MTT $(5.0 \mathrm{mg} / \mathrm{mL})$ solution in PBS was added to each well and incubated for another $5 \mathrm{~h}$, and the medium was replaced with $150 \mu \mathrm{L}$ of DMSO. After $30 \mathrm{~min}$. the optical density (OD) at $490 \mathrm{~nm}$ was measured using a microplate reader (Synergy 4, BioTek, U.S.A). The relative cell viability was defined as: Cell viability $(\%)=\left(\mathrm{OD}_{49(\text { sample) }} / \mathrm{OD}_{490(\text { control })}\right) \times 100 \%$, where $\mathrm{OD}_{490(\text { control) }}$ and $\mathrm{OD}_{490(\text { sample) }}$ were obtained in the absence/presence of samples respectively. Data were shown as mean \pm standard deviation (SD) based on three independent measurements.

\subsection{PI and DAPI co-staining assay}

PC12 cells were seeded at the confocal cell culture dish in DMEM containing $10 \%$ FBS and incubated at $37{ }^{\circ} \mathrm{C}$ for $24 \mathrm{~h}$. After the cells were treated with the $\mathrm{A} \beta_{140}$ growth solution or other compounds, the cell culture medium was replaced with the fresh medium containing PI (10 $\mu \mathrm{L}, 10 \mu \mathrm{g} / \mathrm{mL}$ ). After $30 \mathrm{~min}$, the culture medium was removed and washed three times with PBS. To stain the nucleus of the cell, the cell was further incubated with the cell culture medium with DAPI $(100 \mu \mathrm{L}$, $5 \mu \mathrm{g} / \mathrm{mL}$ ) for $5 \mathrm{~min}$ at $37^{\circ} \mathrm{C}$. Then, the cells were photographed by a Nikon laser scanning confocal microscopy (A1R-CF, Nikon, Japan).

\section{Results and discussion}

\subsection{Fabrication of DA-, L-Phe- and L-Tyr-functionalized USGNPs}

So far, several kinds of nanomaterials have presented the capability of regulating $\mathrm{A} \beta$ peptide fibrillation in vitro by either influencing the conformation of the monomer or delaying the nucleation process. ${ }^{26}$ The size is typically tens of nanometer. Since AD takes place inside the brain, the potential capability of crossing the BBB should be an essential requirement for the design of therapeutic agent. Earlier experimental results have verified that the BBB crossing efficiency can be greatly improved when the size of nanoparticles is small enough. ${ }^{36,37,40}$ For instance, Huang et al. demonstrated that ultra-small-sized nanoparticles $(<10 \mathrm{~nm})$ exert certain advantages over larger ones in terms of localization and penetration of brain tumors in mice. ${ }^{41}$ On this basis, USGNPs were synthesized. The UV-Vis absorption spectrum of freshly synthesized USGNPs is shown in Fig. 1a (the left column). Characteristic single and narrow absorption peak at around $516 \mathrm{~nm}$ is readily observed. The TEM measurements (the right column in Fig. 1a) demonstrated that the size of these USGNPs is $4.1 \pm 0.7 \mathrm{~nm}$, which is well in accordance with the hydrodynamic size distribution in solution $(6.9 \pm 0.5 \mathrm{~nm}$, the DLS measurements).

Generally, to confer functional molecules onto the surface of nanoparticles, physical adsorption and chemical conjugation are the commonly adopted two strategies. In contrast to physical association, covalent chemical conjugation is more attractive because of the good stability under physiological surroundings. ${ }^{42}$ In this regard, DA, L-Phe and L-Tyr were firstly crosslinked with thioglycolic acid through the linker of EDC/NHS. According to the gold/thiol chemistry, these compounds can be specifically functionalized onto the nanoparticle surface. The conjugation process was verified with FT-IR spectroscopy. As illustrated in Fig. 1e, the FT-IR spectra of DA-, L-Tyr- and L-PheUSGNPs were colored with blue, black and red respectively, while the spectrum of blank USGNPs was marked with green. For DA-USGNPs, the peaks at 1646,1558 , and $1218 \mathrm{~cm}^{-1}$ are attributed to the stretching vibrations of $\mathrm{C}=\mathrm{O}$ (amide I), formation vibration of $\mathrm{N}-\mathrm{H}$ (amide II) and stretching vibrations of $\mathrm{C}-\mathrm{N}$ (amide III) respectively. These peaks manifest the presence of amide. To be specific, the appearance of these 
(a)
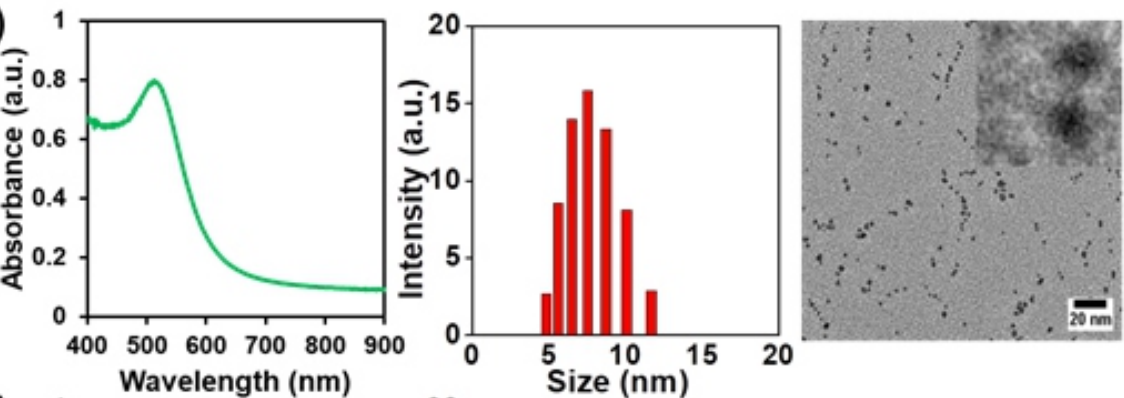

(b)
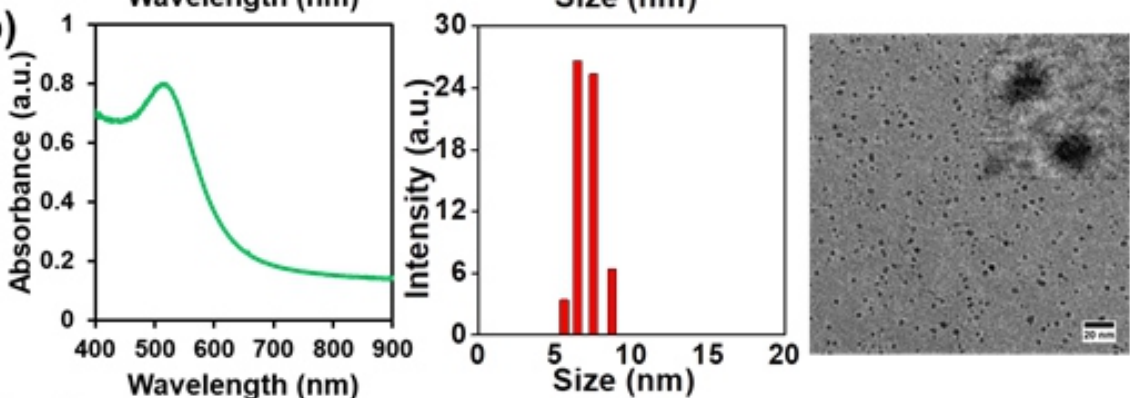

(c)
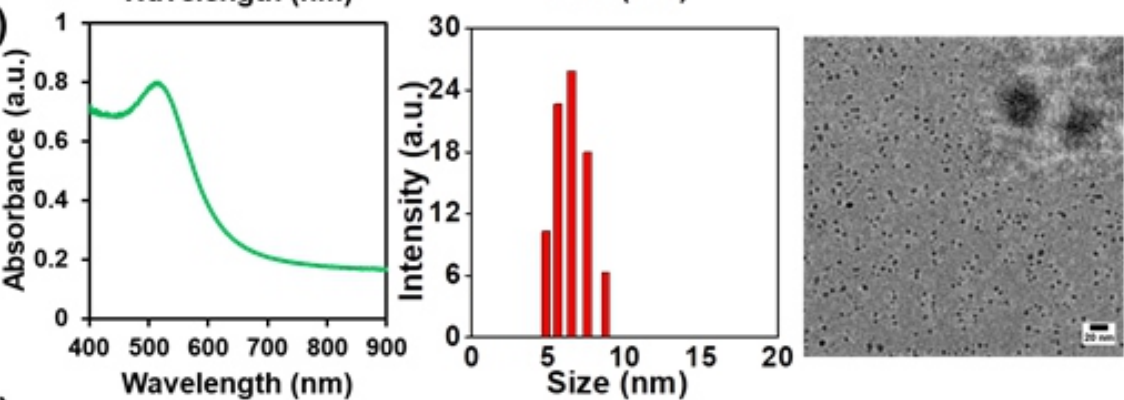

(d)
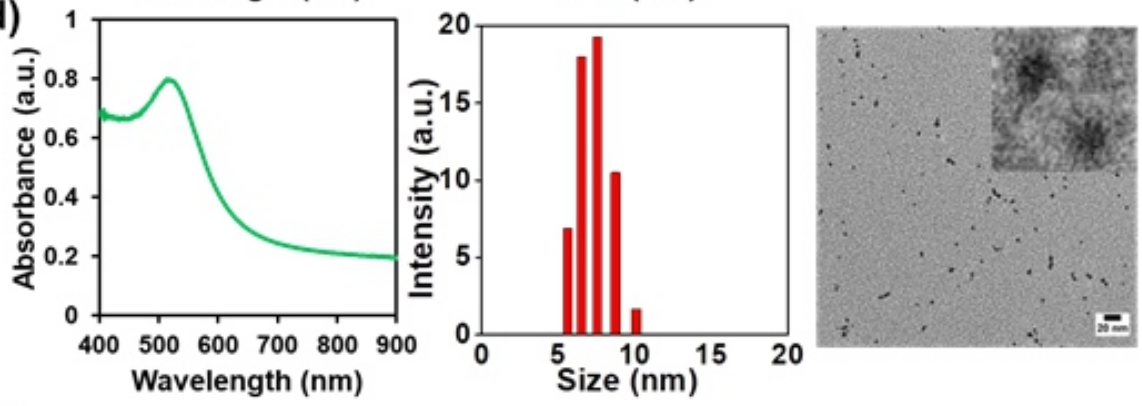

(e)

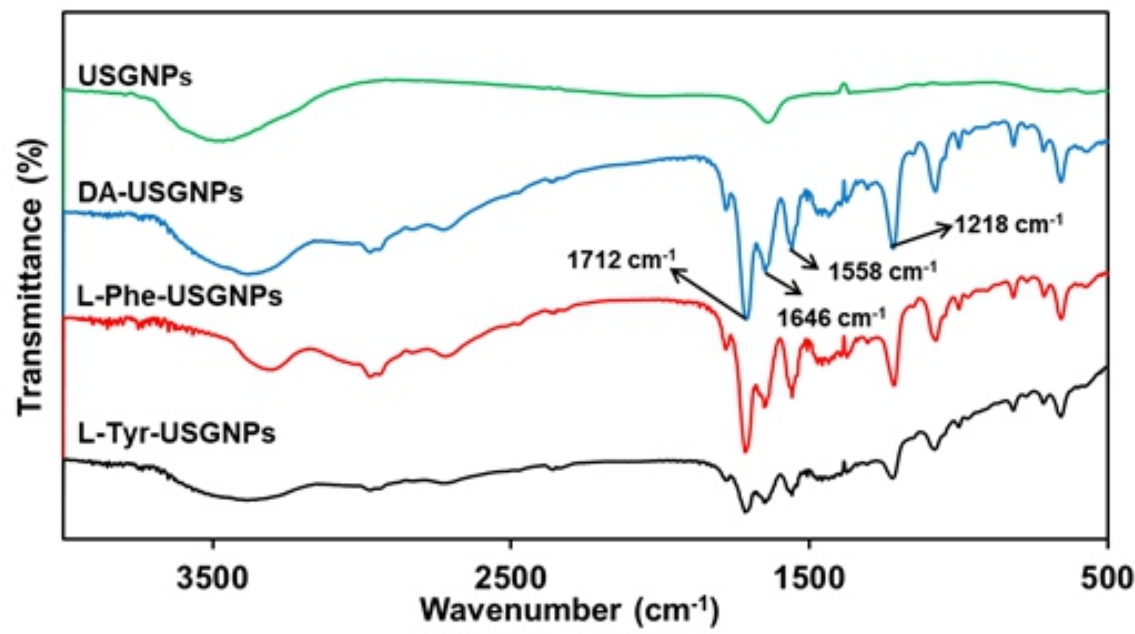

Fig. 1 UV-Vis absorption spectra (left), hydrodynamic size distributions (middle) and TEM images of bare USGNPs (a), DA-USGNPs (b), L-PheUSGNPs (c) and L-Tyr-USGNPs (d). (e) The FT-IR spectra of bare USGNPs (green), DA-USGNPs (blue), L-Phe-USGNPs (red) and L-Tyr-USGNPs (black) respectively. 
peaks indicates the successful combination of $\mathrm{HS}-\mathrm{CH}_{2}-\mathrm{COOH}$ with DA, while they cannot be found in blank USGNPs. In addition, the functionalized DA could be further modified onto the surface of USGNPs with the aid of Au-SH bond. The peak at $1712 \mathrm{~cm}^{-1}$ can be ascribed to the stretching vibrations of $\mathrm{C}=\mathrm{O}$ from the carboxyl group and the broad absorption bands from $3150-3400 \mathrm{~cm}^{-1}$ indicate the presence of amino $\left(-\mathrm{NH}_{2}\right)$ and hydroxyl $(-\mathrm{OH})$ functional groups. Similarly, the peaks noted above were also observed from the other two functionalized USGNPs, demonstrating the successful conjugation of functional molecules on the surface of USGNPs. In addition, zeta potential measurements were performed to confirm the conjugation process. As shown in Table 1, without the modification process, the freshly synthesized USGNPs exhibited strong negative charge. After that, the zeta potential of functionalized USGNPs was greatly increased but still exhibited slight negative charge, further confirming a successful conjugation process. As verified by the TEM and DLS measurements, the USGNPs are monodispersed in solution after the conjugation process as shown in Fig. 1.

3.2 Modulation of $\mathrm{A} \boldsymbol{\beta}_{1-40}$ fibrillation by DA-, L-Phe- and L-Tyrfunctionalized USGNPs

Prior to modulate the self-assembly process, we firstly explored the growth kinetics of $\mathrm{A} \beta_{140}$ in $\mathrm{PB}$ solution at $37{ }^{\circ} \mathrm{C}$. Under this condition, the monomer peptide could gradually self-assemble into oligomers and then form fibrils. By labeling these aggregated peptides with ThT (which can specifically bind to $\beta$-sheet structure of $\mathrm{A} \beta$ fibril and then result in intense fluorescence from the aggregations), the oligomers, seeds and fibrils can be measured by fluorescence spectroscopy or directly observed by fluorescence microscopy. ${ }^{24,43}$ As shown in Fig. 2, initially, only individual diffraction-limited bright spots appeared in the fluorescence microscopic image, which are basically ascribed to aggregated oligomers in the stock solution. As time goes on, the peptides were gradually assembled together and some elongated fibrils appeared in the fluorescence microscopic image. The majorities of them were dispersed separately and exhibited in a rod shape. Further extending the incubation time, the length of the fibrils was increased gradually and followed with slight bending along the longitudinal direction.

To explore the effect of functionalized USGNPs on the $\mathrm{A} \beta_{140}$ self-assembly process, these particles were mixed with $\mathrm{A} \beta_{140}$ monomer solution together under the same culture condition respectively. As shown in Fig. 3, only few diffraction-limited bright spots appeared in the fluorescence microscopic image in the beginning. This is due to the pre-formed oligomers or proto-fibrils in the peptide stock solution. $2 \mathrm{~h}$ later, elongated fibril was not observed in the sample treated with DA-USGNPs in contrast to the control without the addition of functionalized USGNPs. Even $4 \mathrm{~h}$ later, only few seed fibrils appeared in the fluorescence microscopic image with length comparable to the

Table 1 The Zeta potential measurements.

\begin{tabular}{ccccc}
\hline Name & USGNPs & DA-USGNPs & L-Phe-USGNPs & L-Tyr-USGNPs \\
\hline Zeta & $-58.4 \pm 3.5$ & $-14.9 \pm 2.1$ & $-16.5 \pm 1.9$ & $-18.9 \pm 2.5$ \\
potential/mV & & & & \\
\hline
\end{tabular}

(a)

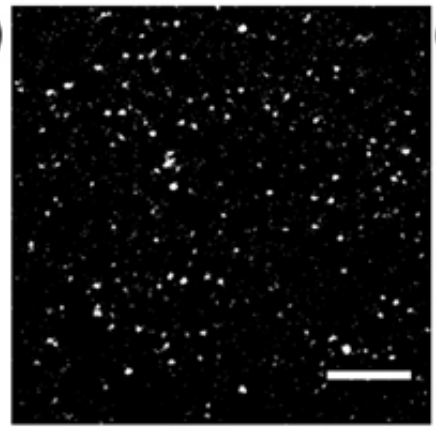

$\mathbf{O h}$

(d)

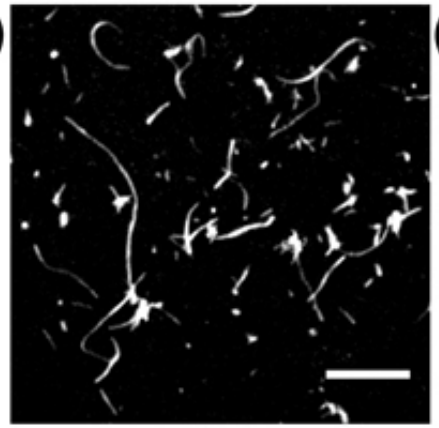

$5 \mathrm{~h}$

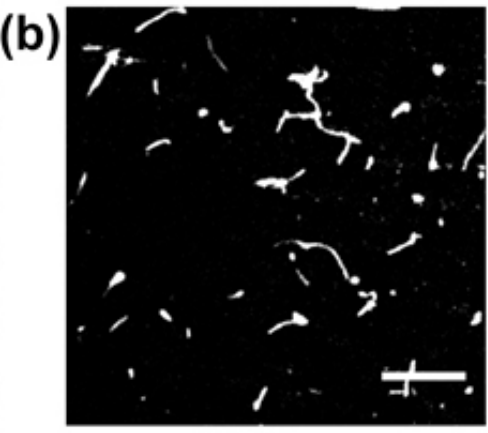

$2 \mathrm{~h}$

(e)

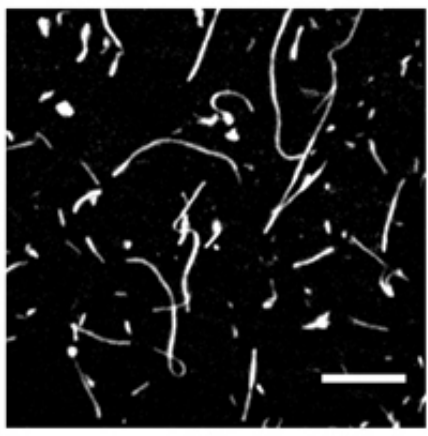

$12 \mathrm{~h}$

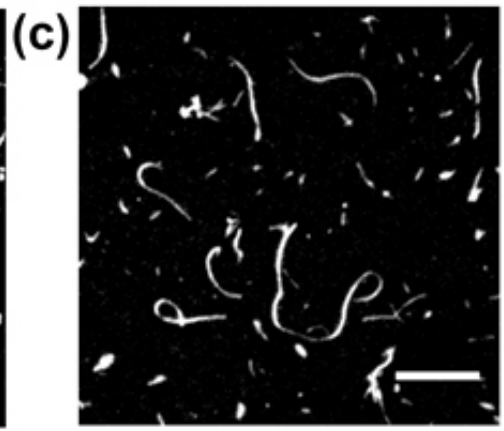

$4 \mathrm{~h}$

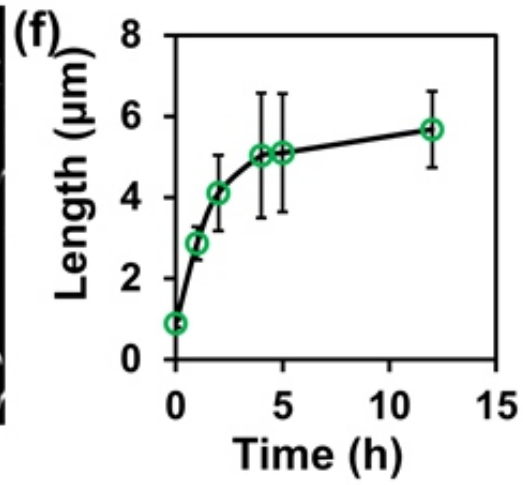

Fig. 2 (a-e) Time-lapse (0-12 h) fluorescence microscopic images of the amyloid peptide fibrillation process in $\mathrm{PB}$ at $37^{\circ} \mathrm{C}$, scale bar $8 \mu \mathrm{m}$. (f) The plot of the fibril length as a function of the incubation time. 
diffraction-limit of optical microscope. These results clearly demonstrated that DA-USGNPs with the final concentration of $43.6 \mathrm{nM}$ could effectively disturb the $\mathrm{A} \beta_{140}$ aggregation. In addition to the microscopic characterizations, the inhibition process can be confirmed by the spectroscopic measurements as shown in Fig. 4a. Interestingly, L-Phe- and L-Tyr-USGNPs also exhibited comparable inhibition effect on the fibrillation process, as shown in Fig. 4b-c. Some studies have demonstrated that the dosage of particles in the solution can influence the modulation mode in the $\mathrm{A} \beta$ aggregation process. ${ }^{26,44}$ For instance, Cabaleirolago et al. observed the striking bifacial effect of the aminemodified polystyrene nanoparticles on $\mathrm{A} \beta$ fibrillation kinetics. ${ }^{24}$ Specifically, the fibrillation process is accelerated by nanoparticles at low particle concentration, while it is retarded at high particle concentration. Based on these considerations, different concentrations of functionalized USGNPs $(14.6,29.0$ and $43.6 \mathrm{nM})$ were used to explore the effect on the $\mathrm{A} \beta_{140}$ fibrillation process. From the spectroscopic results, Fig. 4a-c, no acceleration effect was observed when the concentration of the nanoparticles is far below the point with noticeable inhibition effect. As the concentration of the particles gradually increases, the inhibition effect is more obvious, manifesting the concentration dependent inhibition effect of functionalized USGNPs on $\mathrm{A} \beta_{140}$ fibril growth.

On the consideration of the excellent inhibition effect from the functionalized USGNPs, an essential question is that can USGNPs or DA (and its precursors) itself play analogous inhibition effect on the fibrillation process? In order to address this concern, we performed control experiments through incubating $\mathrm{A} \beta_{140}$ with bare USGNPs (43.6 $\mathrm{nM})$, DA, L-Phe and L-Tyr $(1.53 \mu \mathrm{M}$, the concentration used to conjugate the USGNPs) respectively. As illustrated in Fig. 3, similar to the above observations, initially, only individual sub-diffraction-limited bright spots appeared in the fluorescence image. Distinct from functionalized USGNPs, $2 \mathrm{~h}$ latter, some short fibrils gradually appeared in these control experiments. Further extending the incubation time to 5 $\mathrm{h}$, noticeable fibrils were displayed. The length is slightly shorter than that of blank control. These control experiments demonstrated that DA, L-Phe, L-Tyr and bare USGNPs exhibit trivial inhibition effect on $\mathrm{A} \beta_{140}$ aggregation at short time level. However, as time goes on, certain number of fibrils could still be formed. These phenomena were

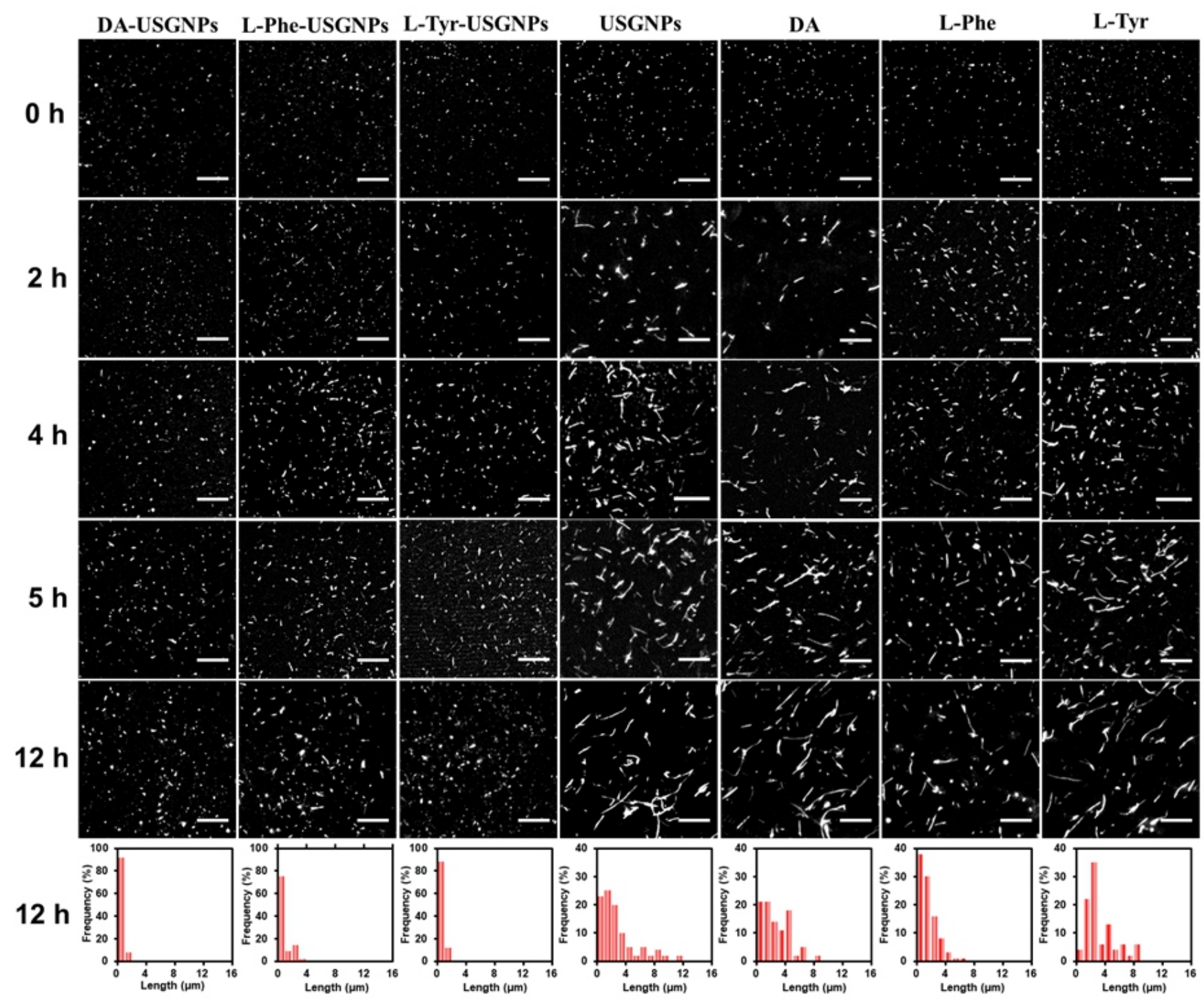

Fig. 3 Fluorescence microscopic images and the length distributions (bottom row) of the resulted amyloid fibrils at different incubation time: 0, 2, 4, 5 and $12 \mathrm{~h}$ after adding DA-USGNPs, L-Phe-USGNPs, L-Tyr-USGNPs, USGNPs, DA, L-Phe and L-Tyr (from left to right) to the A $\beta_{1-40}$ growth solution (with monomer concentration of $50 \mu \mathrm{m}$ ), scale bar $8 \mu \mathrm{m}$. 
(a)

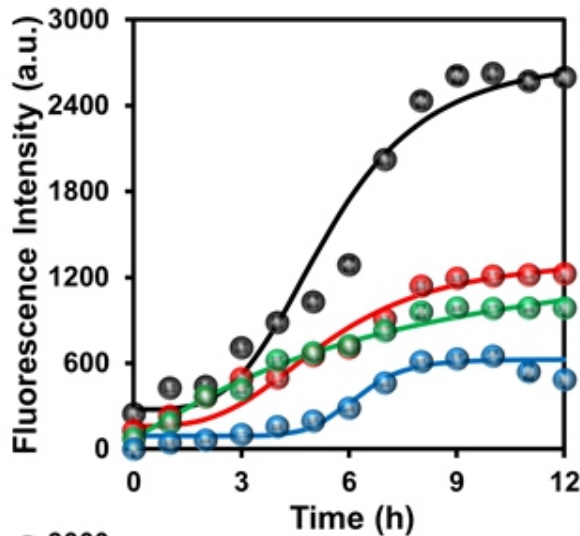

(c)

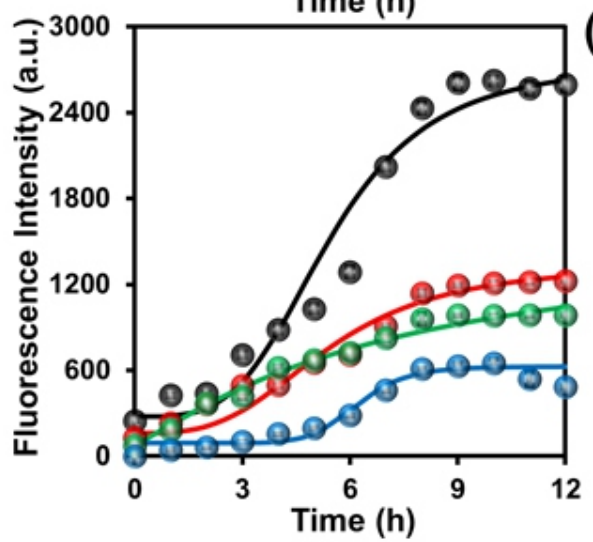

(b)

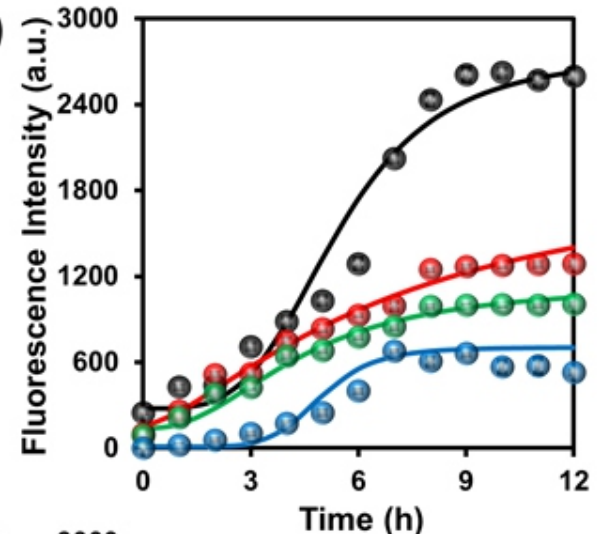

(d)

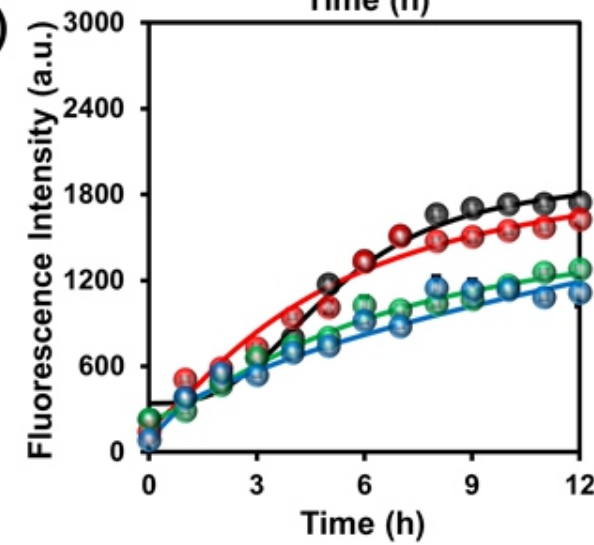

Fig. 4 The solution sample fluorescence intensity measurements. The ThT fluorescence intensity of the $\mathrm{A} \beta_{1-40}$ growth solution in the presence of different concentrations (red $14.6 \mathrm{nM}$, green $29.0 \mathrm{nM}$, and blue $43.6 \mathrm{nM}$ ) of (a) DA-USGNPs, (b) L-Phe-USGNPs, and (c) L-Tyr-USGNPs as a function of incubation time. Black dotted line is the control without the addition of functionalized USGNPs. (d) The effect of bare USGNPs (43.6 nM, green), DA

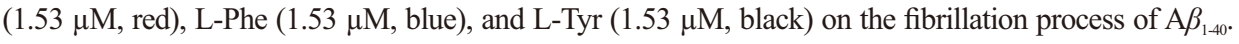

(a)

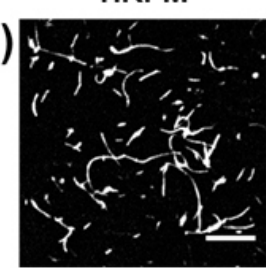

(b)

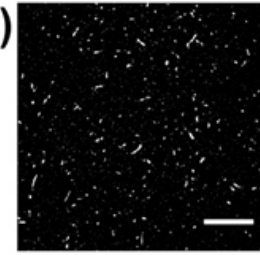

(c)

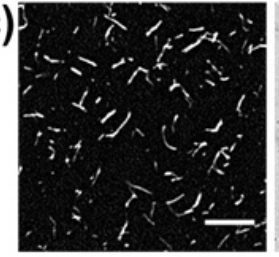

TEM
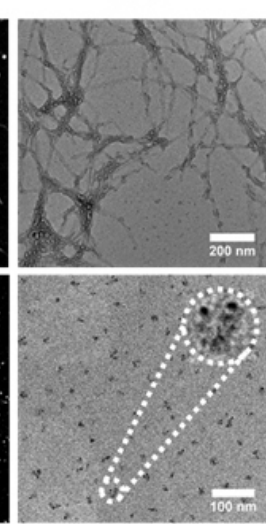

Frequency Histogram
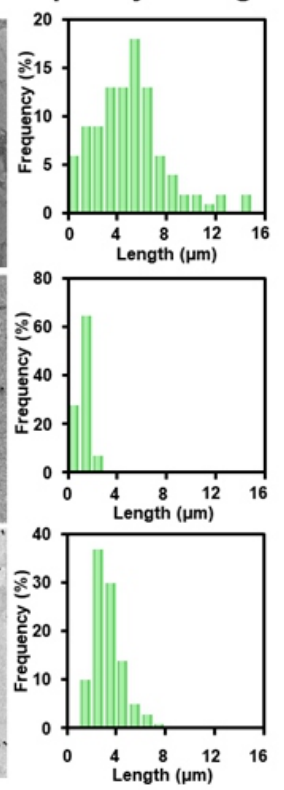

(d)

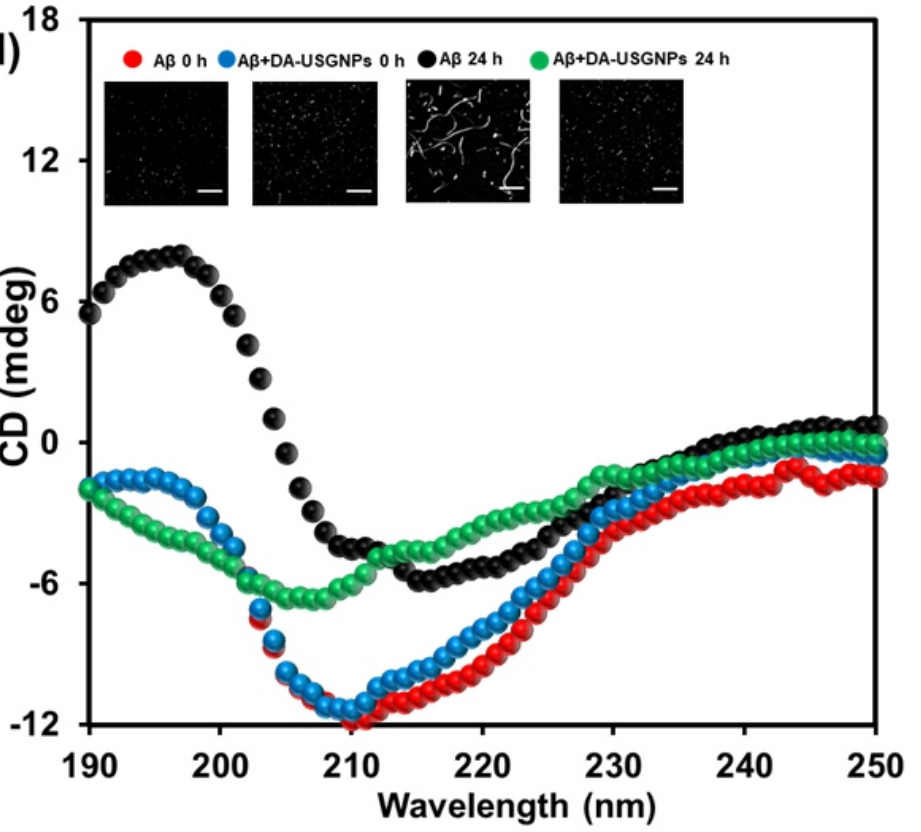

Fig. 5 The fluorescence microscopic images (left), TEM images (middle) and the length distributions (right) of $A \beta_{140}$ fibrils determined from the corresponding fluorescent microscopic images of $\mathrm{A} \beta_{140}$ peptides growth solution after incubating at $37^{\circ} \mathrm{C}$ for $12 \mathrm{~h}$ in the presence of (a) $0 \mathrm{nM}$, (b) 43.6 nM of DA-USGNPs. (c) The fluorescence microscopic and TEM characterizations of preformed A $\beta_{140}$ fibrils stained by DA-USGNPs. The scale bar for TIRFM is $8 \mu \mathrm{m}$. (d) The CD spectroscopic characterizations of $\mathrm{A} \beta_{140}$ peptides growth solution at $0 \mathrm{~h}$ (red), at $24 \mathrm{~h}$ (black), the mixture of $\mathrm{A} \beta_{140}$ peptides growth solution with DA-USGNPs at $0 \mathrm{~h}$ (blue), and the mixture of $\mathrm{A} \beta_{140}$ peptides growth solution with DA-USGNPs at $24 \mathrm{~h}$ (green). 
confirmed by the ensemble spectroscopic measurements as shown in Fig. 4d. This kind of inhibition might be due to the delayed seed formation process.

\subsection{Understanding the interactions between $\mathbf{A} \boldsymbol{\beta}_{1-40}$ and functionalized USGNPs}

In order to understand the inhibition mechanism of functionalized USGNPs, we then performed TEM measurements to decipher the morphological information of the resulted $\mathrm{A} \beta_{1-40}$ aggregates. As illustrated in Fig. 5a, the $\mathrm{A} \beta_{1-40}$ fibrils in the blank control were characterized by long and rod structure. However, no fibril appeared after being incubated with DA-USGNPs even for $12 \mathrm{~h}$. What should be mentioned is that only some short seeds with length around $10 \mathrm{~nm}$ were observed in the TEM image after co-incubation of DA-USGNPs with $\mathrm{A} \beta_{1-40}$ for $12 \mathrm{~h}$. On further inspection of the TEM image, it is worth to point out that all of the seed fibrils are covered with DA-USGNPs selectively. No discernable free DA-USGNPs were noted in Fig. 5b, indicative of a high affinity of DA-USGNPs toward the seed fibrils. To further confirm this argument, we also co-incubated the pre-formed fibrils with DA-USGNPs together for $10 \mathrm{~min}$ and then imaged with TEM. As shown in Fig. 5c, the DA-USGNPs indeed attach on the backbone of the preformed fibrils. Because of the strong interaction with $\mathrm{A} \beta_{1-40}$, it should be difficult for those monomers or oligomers to assemble onto the growth point of seed fibrils, resulting in an efficient inhibition effect.

Earlier explorations have demonstrated that plenty of nanoparticles (NPs), as the efficient modulators, have displayed size and concentration dependent inhibition, acceleration and dual effects on the self-assembly process due to the case-specific interaction (hydrophobic, electrostatic, hydrogen bond, covalent bond and van der Waals forces) between the $\mathrm{A} \beta_{140}$ individual parts and NPs. In general, NPs possess enormous surface areas and powerful adsorption capacity will result in the high local concentration of $\mathrm{A} \beta_{140}$ on the NPs surface and then accelerate the $\mathrm{A} \beta_{140}$ aggregation. Nevertheless, the active sites located at the $\mathrm{A} \beta_{140}$ critical sequence which might be helpful for fibrillation could be blocked and the $\mathrm{A} \beta_{140}$ concentration in solution could also be reduced after the adsorption process, leading to the effective inhibition on the fibrillation. The competition between these two factors would ultimately generate different effects on $\mathrm{A} \beta_{140}$ aggregation. ${ }^{23,24,27,45,46}$

Besides the morphological characterizations, it is also essential to understand the molecular interactions between functionalized USGNPs and $\mathrm{A} \beta_{1-40}$. Previous works have reported that the fibrillation process of $\mathrm{A} \beta_{1-40}$ peptides is basically regulated by two major interaction modes. One is the $\pi-\pi$ interaction between aromatic amino acid residues. The other is the hydrophobic interaction between hydrophobic amino acids. ${ }^{47-53}$ In addition, some explorations have also indicated that the $\mathrm{A} \beta_{1}$. ${ }_{40}$ nucleation is driven by the hydrophobic interactions due to the fact that hydrophobic nanoparticles can only interfere the species within the nucleation phase, and the succeeding elongation is propelled by electrostatic interactions and hydrogen bonding that can be interfered by the negatively charged nanoparticles or redirected due to the specific interaction with the negatively charged nanoparticles. ${ }^{22-24}$ As a control, functional molecules and pure USGNPs display analogous inhibitory ability on the $\mathrm{A} \beta_{140}$ nucleation at the initial stage, while the latter manifest more prominent inhibition effect on the elongation process. These results could be interpreted by the following two points: (a) the hydrophobic interaction between $\mathrm{A} \beta_{140}$ and functional molecules due to their hydrophobic nature of the benzyl side chain leads to their inhibitory effect on $\mathrm{A} \beta_{140}$ nucleation. The adsorption of $\mathrm{A} \beta_{140}$ monomer and oligomer on the USGNPs surface can impede the $\mathrm{A} \beta_{140}$ nucleation, because the active sites located at the $\mathrm{A} \beta_{140}$ monomer critical sequence facilitating the fibrillation are blocked as well as the $\mathrm{A} \beta_{1-40}$ concentration in solution is reduced. (b) The superior inhibition effect of functionalized USGNPs on the elongation process should be ascribed to the interaction of short $\mathrm{A} \beta_{1-40}$ seeds with the negatively charged nanoparticles, which could be verified by the TEM image in Fig. 5b. In this case, the functionalized USGNPs effectively attach on the backbone of short $\mathrm{A} \beta_{140}$ seeds. With respect to the functionalized USGNPs, their higher inhibitory ability on $\mathrm{A} \beta_{140}$ nucleation compared with the same concentration molecules in the control experiment Fig. 4 should derive from the enhanced hydrophobic interaction by introducing the hydrophobic molecules onto the surface of USGNPs together with the adsorption of $\mathrm{A} \beta_{1-40}$ monomer. However, these interactions should be benefited from their enormous surface areas. Moreover, Fig. 5b displays that almost all the DA-USGNPs bind with $\mathrm{A} \beta_{140}$ seeds, preventing the formation of longer fibrils.

Furthermore, these speculations were proved on the basis of the conformational switching of $\mathrm{A} \beta_{1-40}$ secondary structure according to the $\mathrm{CD}$ spectroscopy as shown in Fig. 5d. Well-defined $\beta$-sheet structures were formed with the characteristic positive peak at $198 \mathrm{~nm}$ and broad negative band at $215 \mathrm{~nm}$ after incubated the peptide in $\mathrm{PB}$ at $37{ }^{\circ} \mathrm{C}$ for $24 \mathrm{~h}$. However, when $\mathrm{A} \beta_{140}$ was incubated with DA-USGNPs (43.6 nM) at $37{ }^{\circ} \mathrm{C}$ for $24 \mathrm{~h}$, those two typical peaks disappeared, indicative of the scrambling of folded peptide structure. These results confirm that the functionalized USGNPs could effectively disturb the oligomerization and fibrillation process of $\mathrm{A} \beta_{140}$ under favorable concentration.

\subsection{Regulation of the cytotoxic effect of $A \beta_{140}$ with functionalized USGNPs}

In order to corroborate the potential applications of the functionalized USGNPs, their toxicities to the neuronal cells should be explored. On this basis, the cell viability MTT assays, as a colorimetric assay measuring the metabolic activities of mitochondria, were carried out by employing PC12 cells as a model (Fig. 6a). Negligible influence of functionalized USGNPs on the cell viability was noted in the particle concentration range of 14.6-43.6 nM, where efficient fibrillation inhibition effect was observed. Then the cytotoxic effect of $\mathrm{A} \beta_{1-40}$ fibrils was also explored. The $\mathrm{A} \beta_{1-40}$ monomer was firstly incubated in PB for $12 \mathrm{~h}$ at $37^{\circ} \mathrm{C}$ until noticeable fibrils were appeared (Fig. 2d). The cells were co-incubated with the end-point products of $\mathrm{A} \beta_{1-40}$ growth solution for another $24 \mathrm{~h}$ and analyzed by MTT assay as shown in Fig. $6 \mathrm{~b}$. Evidently, around $30 \%$ of the cells were dead after this treatment in contrast to the control without the addition of $\mathrm{A} \beta_{1-40}$ growth solution. On the addition of functionalized USGNPs (with a final concentration of $43.6 \mathrm{nM}$ ), interestingly, around $90 \%$ of the cells still survive even treated the cells with the same dosage of $\mathrm{A} \beta_{1-40}$ growth solution, indicating excellent cell protection effect from the functionalized USGNPs. It is worth to point out that, on the aspect of $\mathrm{A} \beta_{140}$ fibrillation inhibition effect in solution, there is no evident difference among those particles. However, on the consideration of the cell protection effect, slightly improved cell viability was noted from the DA-USGNPs over L-Phe-USGNPs and L-Tyr-USGNPs.

Besides the MTT assay, fluorescence confocal microscopic characterizations were further performed to characterize the cytotoxicity of $\mathrm{A} \beta_{1-40}$ fibrils as well as the cell protection effect from the functionalized USGNPs. In this experiment, propidium iodide (PI, red) was adopted as the indicator for cell apoptosis, which can pass through the damaged cell membrane and stain the nucleus while cannot pass through the living cell membrane, making it is useful to differentiate necrotic, apoptotic and healthy cells. As shown in Fig. 6c, without $\mathrm{A} \beta_{140}$ growth solution treatment, the PI cannot pass through the living cell membrane. No co-localization was observed from the blue channel 

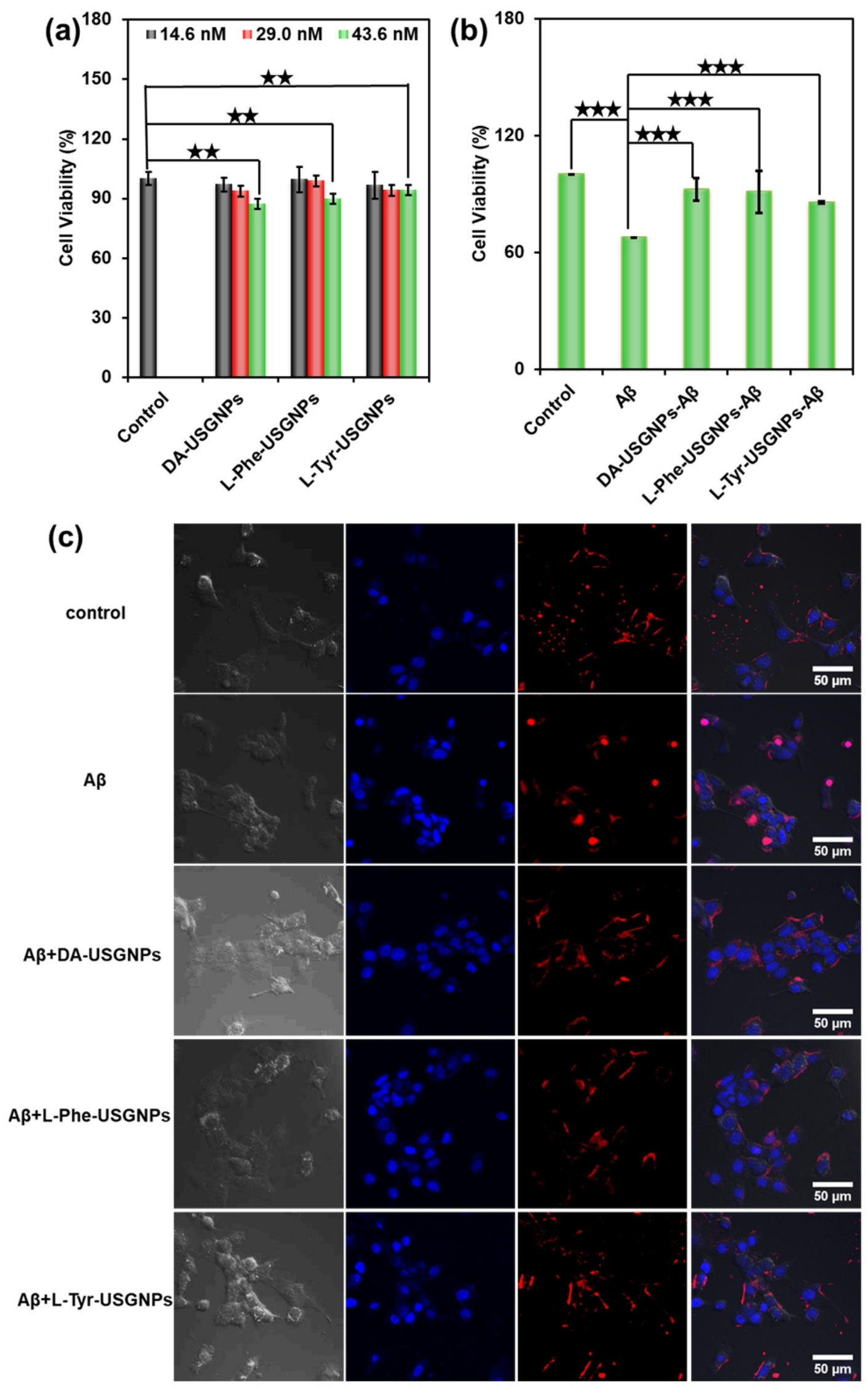

Fig. 6 The cellular cytotoxicity assays (MTT) of (a) different concentrations of functionalized USGNPs, (b) the end-point products (12 h) of A $\beta_{140}$ growth solution alone and the mixture of functionalized USGNPs and $\mathrm{A} \beta_{140}$ growth solution toward PC12 cells. (c) The fluorescence confocal microscopic characterizations of the cytotoxic effect of $\mathrm{A} \beta_{140}$ growth solution without and with functionalized USGNPs toward PC12 cells. From left to right are the bright-field, blue fluorescence channel stained by DAPI, red fluorescence channel stained by PI, and the merged microscopic image. 
stained with 4',6-diamidino-2-phenylindole, (DAPI, blue, nucleus stain) and red channel, demonstrating the good cell viability in the control. In the case of the cells treated by $\mathrm{A} \beta_{1-40}$ growth solution for $24 \mathrm{~h}$, even though the morphology of the cells were still intact, some of the cells were evidently stained by PI in the nucleus as confirmed by the DAPI channel, indicative of the apoptosis induced by the $\mathrm{A} \beta_{1-40}$ fibrils. In agreement with the MTT assay, on the addition of DA-, L-Phe- or LTyr-USGNPs, the cells (treated by $\mathrm{A} \beta_{140}$ growth solution) were only stained on nucleus by DAPI. Analogous to the blank control, no colocalization was noted from the blue and red channels, demonstrating the good cell viability after the addition of functionalized USGNPs.

\section{Conclusions}

In conclusion, in this work, USGNPs with diameter of $\sim 4 \mathrm{~nm}$ were synthesized and covalently crosslinked with neurotransmitter DA and its synthetic precursor L-Phe and L-Tyr. The real-time fluorescence spectroscopic and optical microscopic characterizations demonstrated that the $\mathrm{A} \beta_{140}$ fibrillation process in biological milieu can be effectively inhibited by these functionalized USGNPs. The strong and specific interaction with $\mathrm{A} \beta_{140}$ seed fibrils was demonstrated to be the essential mechanism to regulate the fibrillation process, which is corroborated by the TEM and CD spectroscopic characterizations. More importantly, these USGNPs exhibit excellent cell biocompatibility and protection effect toward the cells treated by $\mathrm{A} \beta_{1-40}$ growth solution as confirmed by the ensemble MTT assay and confocal microscopic imaging results. In contrast to previous works, one of the essential merits of these USGNPs is the small size dimension, which might be more suitable for the potential in vivo application. As a consequence, these functionalized USGNPs would shed new light on the exploration of robust strategy for the treatment of $\mathrm{AD}$ in the future.

\section{Conflict of interest}

There are no conflicts to declare.

\section{Acknowledgements}

This work was supported by national natural science foundation of China (NSFC, Project no. 21522502), the Fundamental Research Funds for the Central Universities, the Excellent Youth Scholars of Hunan Provincial Education Department (17B155) and the Opening Fund of Key Laboratory of Chemical Biology and Tradition Chinese Medicine Research (Ministry of Education of China), Hunan Normal University.

\section{References}

1. I. W. Hamley, Chem. Rev., 2012, 112, 5147-5192.

2. J. Hardy and D. J. Selkoe, Science, 2002, 297, 353-356.

3. R. Kayed, E. Head, J. L. Thompson, T. M. McIntire, S. C. Milton, C. W. Cotman and C. G. Glabe, Science, 2003, 300, 486-489.

4. A. Rauk, Chem. Soc. Rev., 2009, 38, 2698-2715.

5. G. Zündorf and G. Reiser, Antioxid. Redox Sign., 2011, 14, 1275-1288.

6. B. S. Diniz, J. A. Pinto, M. L. C. Gonzaga, F. M. Guimarães, W. F. Gattaz and O. V. Forlenza, Eur. Arch. Psychiatry. Clin. Neurosci., 2009, 259, 248256.

7. G. Gao, M. Zhang, D. Gong, R. Chen, X. Hua and T. Sun, Nanoscale, 2017, 9, 4107-4113

8. G. Wei, Z. Su, N. P. Reynolds, P. Arosio, I. W. Hamley, E. Gazitf and R. Mezzenga, Chem. Soc. Rev., 2017, 46, 4661-4708.

9. T. Sylla, L. Pouységu, C. G. Da, D. Deffieux, J. P. Monti and S. Quideau, Angew. Chem. Int. Ed., 2015, 54, 8217-8221.

10.X. Han, J. Park, W. Wu, A. Malagon, L. Wang, E. Vargas, A. Wikramanayake, K. N. Houk and R. M. Leblanc, Chem. Sci., 2017, 8, 20032009 .
11. W. Yang, Y. Wong, O. T. Ng, L. P. Bai, D. W. Kwong, Y. Ke, Z. H. Jiang, H. W. Li, K. K. Yung and M. S. Wong, Angew. Chem. Int. Ed., 2012, 51, 1804 1810.

12. V. V. Shvadchak, K. Afitska and D. A. Yushchenko, Angew. Chem. Int. Ed., 2018, 57, 5690-5694.

13. Q. Luo, Y. X. Lin, P. P. Yang, Y. Wang, G. B. Qi, Z. Y. Qiao, B. N. Li, K Zhang, J. P. Zhang and L. Wang, Nat. Commun., 2018, 9, 1802-1813.

14. Y. Chen, Z. Chen, Y. Sun, J. Lei and G. Wei, Nanoscale, 2018, 10, 8989 8997.

15. I. A. Cavini, C. E. Munte, M. B. Erlach, G. T. Van, I. Kadish, T. Zhang, T. Ziehm, L. Nagel-Steger, J. Kutzsche and W. Kremer, Chem. Commun., 2018, 54, 3294-3297.

16. M. J. Kogan, N. G. Bastus, R. Amigo, D. Grillo-Bosch, E. Araya, A. Turiel, A. Labarta, E. Giralt and V. F. Puntes, Nano Lett., 2006, 6, 110-115.

17. R. C. Petersen, R. G. Thomas, M. Grundman, D. A. Bennett, R. S. Doody, S. H. Ferris, D. Galasko, S. Jin, J. Kaye, A. I. Levey, E. Pfeiffer, M. Sano, C. H. V. Dyck and L. J. Thal, N. Engl. J. Med., 2005, 352, 2379-2388.

18. J. Dong, J. E. Shokes, R. A. Scott and D. G. Lynn, J. Am. Chem. Soc., 2006 128, 3540-3542.

19. A. Kumar, L. Moody, J. F. Olaivar, N. A. Lewis, R. L. Khade, A. A. Holder, Y. Zhang and V. Rangachari, ACS Chem. Neurosci., 2010, 1, 691-701.

20. T. John, A. Gladytz, C. Kubeil, L. L. Martin, H. J. Risselada and B. Abel, Nanoscale, 2018, 10, 20894-20913.

21. Y. D. Álvarez, J. A. Fauerbach, J. V. Pellegrotti, T. M. Jovin, E. A. JaresErijman and F. D. Stefani, Nano Lett., 2013, 13, 6156-6163.

22. Y. H. Liao, Y. J. Chang, Y. Yoshiike, Y. C. Chang and Y.-R. Chen, Small, 2012, 8, 3631-3639.

23. C. Cabaleirolago, F. Quinlanpluck, I. Lynch, S. Lindman, A. M. Minogue, E. Thulin, D. M. Walsh, K. A. Dawson and S. Linse, J. Am. Chem. Soc., 2008, 130, 15437-15443

24. C. Cabaleirolago, F. Quinlanpluck, I. Lynch, K. A. Dawson and S. Linse, ACS Chem. Neurosci., 2010, 1, 279-287.

25. M. Zhang, X. Mao, Y. Yu, C. X. Wang, Y. L. Yang and C. Wang, Adv. Mater. 2013, 25, 3780-3801.

26. L. Xiao, D. Zhao, W. H. Chan, M. M. F. Choi and H. W. Li, Biomaterials, 2010, 31, 91-98

27. C. Cabaleirolago, O. Szczepankiewicz and S. Linse, Langmuir, 2012, 28, 1852-1857.

28. F. U. Amin, A. K. Hoshiar, T. D. Do, Y. Noh, S. A. Shah, M. S. Khan, J. Yoon and M. O. Kim, Nanoscale, 2017, 9, 10619-10632.

29. H. Huang, P. Li, M. Zhang, Y. Yu, Y. Huang, H. Gu, C. Wang and Y. Yang, Nanoscale, 2017, 9, 5044-5048.

30. Y. Liu, L. P. Xu, W. Dai, H. Dong, Y. Wen and X. Zhang, Nanoscale, 2015, 7, 19060-19065.

31. M. Mahmoudi, F. Quinlan-Pluck, M. P. Monopoli, S. Sheibani, H. Vali, K. A. Dawson and I. Lynch, ACS Chem. Neurosci., 2013, 4, 475-485.

32. T. Toneff, L. Funkelstein, C. Mosier, A. Abagyan, M. Ziegler and V. Hook, Peptides, 2013, 46, 126-135.

33. Kerber and A. Kevin, J. Neuro-Ophthalmol., 2008, 28, 365-366.

34. R. A. Mitchell, N. Herrmann and K. L. Lanctôt, CNS Neurosci. Ther, 2011, 17, 411-427.

35. S. Meyers, Altern. Med. Rev., 2000, 5, 64-71.

36. Y. Song, D. Du, L. Li, J. Xu, P. Dutta and Y. Lin, ACS Appl. Mater. Interfaces, 2017, 13, 20410-20416.

37. D. Liu, B. Lin, W. Shao, Z. Zhu, T. Ji and C. Yang, ACS Appl. Mater Interfaces, 2014, 6, 2131-2136.

38. T. Yin, W. Xie, J. Sun, L. Yang and J. Liu, ACS Appl. Mater. Interfaces, 2016, 8, 19291-19302.

39. F. J. van Spronsen, M. Hoeksma and D.-J. Reijngoud, J. Inherit. Metab. Dis., 2009, 32, 46-51.

40. S. Krol, R. Macrez, F. Docagne, G. Defer, S. Laurent, M. Rahman, M. J. Hajipour, P. G. Kehoe and M. Mahmoudi, Chem. Rev., 2013, 113, $1877-$ 1903.

41. K. Huang, H. Ma, J. Liu, S. Huo, A. Kumar, T. Wei, X. Zhang, S. Jin, Y. Gan, P. C. Wang, S. He, X. Zhang and X. J. Liang, ACS Nano, 2012, 6, 4483-4493.

42. M. Jedrzejczak-Silicka, K. Urbas, E. Mijowska and R. Rakoczy, J. Alloy. Compd., 2017, 709, 112-124. 
43. M. Biancalana and S. Koide, Biochim. Biophys. Acta., 2010, 1804, 14051412 .

44. S. I. Yoo, M. Yang, J. R. Brender, V. Subramanian, K. Sun, N. E. Joo, S. H. Jeong, A. Ramamoorthy and N. A. Kotov, Angew. Chem. Int. Ed., 2011, 50, 5110-5115.

45. R. Vacha, S. Linse and M. Lund, J. Am. Chem. Soc., 2014, 136, 1177611782.

46. Y. Song, E. G. Moore, Y. Guo and J. S. Moore, J. Am. Chem. Soc., 2017, 139, 4298-4301.

47. I. Roterman, M. KrUl, M. Nowak, L. Konieczny, J. Rybarska, B. Stopa, B. Piekarska and G. Zemanek, Med. Sci. Monit., 2001, 7, 771-784.

48. C. M. Dobson, Trends Biochem. Sci., 1999, 24, 329-332.
49. J. Zhou, X. Du, Y. Gao, J. Shi and B. Xu, J. Am. Chem. Soc., 2014, 136, 2970-2973.

50. J. Li, X. Du, S. Hashim, A. Shy and B. Xu, J. Am. Chem. Soc., 2017, 139, 71-74.

51. R. Haburcak, J. Shi, X. Du, D. Yuan and B. Xu, J. Am. Chem. Soc., 2016, 138, 15397-15404.

52. Y. Liang, D. G. Lynn and K. M. Berland, J. Am. Chem. Soc., 2010, 132, 6306-6308.

53. M. C. Hsieh, C. Liang, A. K. Mehta, D. G. Lynn and M. A. Grover, J. Am. Chem. Soc., 2017, 139, 17007-17010.

Publisher's Note Engineered Science Publisher remains neutral with regard to jurisdictional claims in published maps and institutional affiliations. 